Almut Balleer, Britta Gehrke, Brigitte Hochmuth und Christian Merkl*

\title{
Autonomes Fahren statt Stop and Go: Vorschläge zur effektiven Gestaltung der deutschen Kurzarbeit
}

https://doi.org/10.1515/zfwp-2019-2022

Abstract: This article argues that short-time work stabilized employment in Germany substantially during the Great Recession in 2008/09. The labor market instrument acted in timely manner, as it was used in a rule-based fashion. In addition, discretionary extensions were effective due to their interaction with the business cycle. To ensure that short-time work will be effective in the future, this article proposes an automatic facilitation of the access to short-time work in severe recessions. This reduces the likelihood of a too extensive use at the wrong point in time as well as structural instead of cyclical interventions.

\section{Einführung}

Unser Beitrag argumentiert, dass Kurzarbeit in der Großen Rezession der Jahre 2008/2009 einen nennenswerten Beitrag zur Stabilisierung der Beschäftigung erbracht hat. Die hohe Effektivität ist auch darauf zurückzuführen, dass der Zugang zur Kurzarbeit regelgebunden funktioniert und diskretionäre Erleichterungen dieses Arbeitsmarktinstruments in der Großen Rezession mit der konjunkturellen Entwicklung passend interagiert haben. Die derzeitigen Vorschläge aus dem Bundesministerium für Arbeit und Soziales (BMAS), Kurzarbeit per Verord-

\footnotetext{
*Kontakt: Christian Merkl, Universität Erlangen - Nürnberg, Lehrstuhl für Makroökonomik, Lange Gasse 20, 90403 Nürnberg, E-Mail: christian.merkl@fau.de

Almut Balleer, RWTH Aachen, Lehr- und Forschungsgebiet Empirische Wirtschaftsforschung, Templergraben 64, 52062 Aachen, E-Mail: balleer@ewifo.rwth-aachen.de

Britta Gehrke, Universität Erlangen-Nürnberg und Institut für Arbeitsmarkt- und Berufsforschung (IAB), Juniorprofessur für Makroökonomik und Arbeitsmarktforschung, Lange Gasse 20, 90403 Nürnberg, E-Mail: britta.gehrke@fau.de

Brigitte Hochmuth, Universität Erlangen - Nürnberg, Lehrstuhl für Makroökonomik, Lange Gasse 20, 90403 Nürnberg, E-Mail: brigitte.hochmuth@fau.de
} 
nungsermächtigung in Rezessionen schnell diskretionär zu erleichtern, bergen allerdings zwei Gefahren. Erstens könnte der Zugang zu Kurzarbeit zum falschen Zeitpunkt ausgeweitet werden und zweitens wird das Instrument möglicherweise strukturell eingesetzt. Beides könnte zu negativen volkswirtschaftlichen Effekten führen. Wir schlagen dagegen vor, dass der Zugang zur Kurzarbeit regelgebunden in schweren Rezessionen erleichtert wird, um die makroökonomische Effektivität des Instrumentes zu erhöhen. Der erleichterte Zugang könnte sich an entsprechenden wirtschaftlichen Frühindikatoren orientieren.

\section{Kurzarbeit in Deutschland}

Kurzarbeit existiert in Deutschland seit Mitte der 1970er Jahre und kann von Firmen bei der Bundesagentur für Arbeit jederzeit beantragt werden; es wird dabei zwischen konjunktureller, saisonaler und Transfer-Kurzarbeit unterschieden. Das konjunkturelle Kurzarbeitergeld wird gewährt, wenn in Betrieben oder Betriebsabteilungen die regelmäßige betriebsübliche wöchentliche Arbeitszeit infolge wirtschaftlicher Ursachen oder eines unabwendbaren Ereignisses vorübergehend verkürzt wird. Das Kurzarbeitergeld kompensiert dann den Lohn für die ausfallenden Arbeitsstunden zumindest teilweise, normalerweise auf dem Niveau des Arbeitslosengeldes. Kurzarbeit kann daher als ein Teil, bzw. eine Vorstufe der Arbeitslosenversicherung betrachtet werden mit dem Ziel Arbeitslosigkeit zu vermeiden. Da Arbeitszeitkürzungen finanziell nicht vollständig aufgefangen werden, ist die Zustimmung des Betriebsrates bei der Antragstellung nötig. Die Unternehmen beteiligen sich an den Kosten, indem die Sozialversicherungsbeiträge von den Firmen im Regelfall für die gesamte reguläre Arbeitszeit gezahlt werden. ${ }^{1}$ Abbildung 1 zeigt, dass die Nutzung von Kurzarbeit stark über den Konjunkturzyklus schwankt. Im Jahr 2009 ging das deutsche Bruttoinlandsprodukt um mehr als 5 Prozent zurück, während Beschäftigung und Arbeitslosigkeit fast unverändert blieben. Gleichzeitig arbeiteten im Maximum rund 1,5 Millionen Beschäftigte in Kurzarbeit. Auf Basis dieser Beobachtungen wird Kurzarbeit oftmals als ein wesentlicher Faktor für die stabile Entwicklung auf dem deutschen Arbeitsmarkt während der Großen Rezession der Jahre 2008 und 2009 betrachtet. Neben der Großen Rezession wurde Kurzarbeit während der

1 Damit ein Betrieb Kurzarbeitergeld beantragen kann, muss ein erheblicher Arbeitsausfall mit Entgeltausfall vorliegen. Die Voraussetzungen dafür sind erfüllt, wenn der Arbeitsausfall mindestens ein Drittel der Beschäftigten betrifft, aus wirtschaftlichen Gründen oder wegen eines unabwendbaren Ereignisses entsteht, vorübergehend und nicht vermeidbar ist (§ 95 ff. SGB III). 


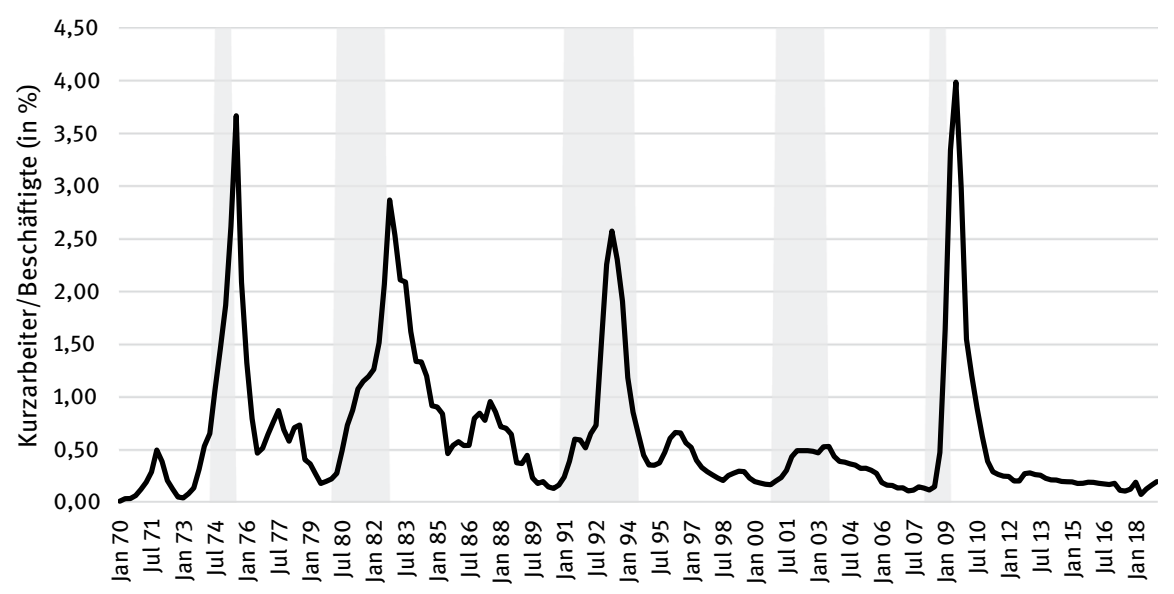

Abbildung 1: Anteil der Kurzarbeiter*innen an der Anzahl der sozialversicherungspflichtigen Beschäftigten in Deutschland.

Datenquelle: Statistik der Bundesagentur für Arbeit, alle Daten sind saisonal bereinigt. Der Datenbruch durch die Wiedervereinigung in der Reihe der sozialversicherungspflichtigen Beschäftigten wurde mit einer Dummy-Schätzung bereinigt. Die grau-schattierten Flächen stellen Rezessionsperioden nach Definition des Economic Cycle Research Institute (ECRI) dar.

ersten und zweiten Ölpreiskrise intensiv genutzt. ${ }^{2}$ Die starke Nutzung der Kurzarbeit während der Ölpreiskrisen (bei einem gleichzeitigen Anstieg der Arbeitslosigkeit) stellt in Frage, ob Kurzarbeit die alleinige Ursache der Arbeitsmarktentwicklung während der Großen Rezession sein kann.

Ähnlich wie beim Steuersystem ist es für die Analyse der makroökonomischen Stabilisierungswirkungen der Kurzarbeit wichtig, die regelgebundene und diskretionäre Komponente zu unterscheiden. Unternehmen, die mit einem Rückgang der Nachfrage konfrontiert sind, können - unabhängig von der konjunkturellen Lage - jederzeit Kurzarbeit bei der Bundesagentur für Arbeit beantragen. Dadurch, dass mehr Unternehmen in einer Rezession in finanzielle Schwierigkeiten geraten, qualifizieren sich diese automatisch für die Nutzung des Instruments (ohne Änderung der Zugangsvoraussetzungen). Wir bezeichnen das als die regelgebundene Komponente von Kurzarbeit. ${ }^{3}$ Darüber hinaus unternehmen die Bundesregierung und die Bundesagentur für Arbeit insbesondere in Rezessionen teilweise weitere Anstrengungen, um die Nutzung von Kurzarbeit auszuweiten

2 Kurz nach der deutschen Wiedervereinigung wurde vor allem Transfer-Kurzarbeit eingesetzt. 3 Im deutschen Steuersystem wäre das analog der automatische Rückgang der Lohn- und Einkommensteuer (bei unverändertem Steuersystem) aufgrund gefallener Bruttoeinkommen in der Rezession. 
(z.B. die Übernahme eines größeren Anteils der Sozialversicherungsbeiträge seitens der öffentlichen Hand, eine Verlängerung der Bezugsdauer, eine weniger strenge Interpretation existierender Regeln zur Kurzarbeitsnutzung oder aktive Bewerbung des Instrumentes). Wir bezeichnen diese ad-hoc Politikmaßnahmen als die diskretionäre Komponente von Kurzarbeit.

\section{Regelgebunde Komponente der Kurzarbeit als automatischer Stabilisator}

Arbeitsmarktentscheidungen sind im Normalfall mittel- bis langfristiger Natur und sind somit teilweise vergleichbar $\mathrm{zu}$ Investitionsentscheidungen. Hierbei werden zwischenzeitlich entstehende Verluste mit Wiedereinstellungskosten und zukünftigem Produktionspotential gegengerechnet. Überwiegen die Verluste, kommt es zu Entlassungen. Kurzarbeit verringert zwischenzeitlich entstehende Verluste und verhindert so Entlassungen. Wird dieser Effekt im Rahmen der regelgebundenen Komponente erwartet, steigt der Wert eines Beschäftigungsverhältnisses, was nicht nur zu weniger Entlassungen, sondern auch zu weniger stark fallenden Einstellungen führt.

Anders als beim Steuersystem lassen sich die regelgebundene und die diskretionäre Komponente der Kurzarbeit nicht direkt beobachten, da insbesondere die Interpretation der Zugangsregeln nicht messbar ist. Balleer et al. (2016) verwenden deswegen eine mikroökonometrische Schätzung auf Basis des IABBetriebspanels und schätzen die regelgebundene Komponente anhand der durchschnittlichen Elastizität der Kurzarbeiter^innen auf Umsatzveränderungen in Betrieben. Die Schätzergebnisse werden dann in ein quantitatives makroökonomisches Gleichgewichtsmodell mit Sucharbeitslosigkeit eingespeist. Das makroökonomische Modell erlaubt kontrafaktische Simulationen, z.B. der Entwicklung der Beschäftigung in der Großen Rezession mit und ohne regelgebundener Komponente. Kernergebnis der Modellsimulation ist, dass die regelgebundene Komponente in der Großen Rezession bis zu 450.000 Jobs gesichert hat. Ein Teil des deutschen Erfolgsmodells Kurzarbeit ist daher die langfristige, automatisch stabilisierende Komponente. 


\section{Konjunkturabhängige Effekte der diskretionären Komponente von Kurzarbeit}

In der Großen Rezession haben die Bundesregierung und die Bundesagentur für Arbeit Kurzarbeit diskretionär stark ausgeweitet. ${ }^{4}$ Balleer et al. (2016) weisen in diesem Zusammenhang auf die Gefahr von Mitnahmeeffekten hin. Diese entstehen, wenn Arbeitskräftehortung aus unternehmerischer Sicht sinnvoll ist und die entsprechenden Finanzierungsmöglichkeiten für diese Hortung zur Verfügung stehen. Überraschende Änderungen in der Kurzarbeitspolitik haben dann wenig Einfluss auf die Arbeitslosigkeit, wohl aber auf die Gewinne der Unternehmen.

Gehrke und Hochmuth (2019) zeigen mithilfe eines strukturellen zeitvariierenden Vektorautoregressiven Modells (Smooth Transition VAR), dass die Beschäftigungseffekte der diskretionären Komponente von Kurzarbeit stark von der Konjunktursituation abhängen. Je tiefer die Rezession, desto mehr Arbeitsplätze können durch diskretionäre Maßnahmen erhalten werden. In der Großen Rezession hat die diskretionäre Komponente der Kurzarbeit bis zu 400.000 zusätzliche Jobs gerettet (siehe Gehrke und Hochmuth, 2019). In Expansionen sind die Beschäftigungseffekte hingegen klein und können sogar negativ werden.

Gehrke und Hochmuth (2019) finden, dass Betriebe, die in der Großen Rezession Kurzarbeit nutzten, eher von Kreditbeschränkungen betroffen waren. Da Kurzarbeit wie eine Lohnsubvention wirkt, kann das Instrument dazu beitragen Kreditbeschränkungen aufzulösen oder zu mindern. ${ }^{5}$ In der Großen Rezession waren diskretionäre Maßnahmen deshalb besonders wirksam. Ein weiterer Grund hierfür kann sein, dass Kurzarbeit einen Verlust von Humankapital in Arbeitslosigkeit verhindert und so Hysteresis Effekten entgegenwirkt.

Laut Gehrke und Hochmuth (2019) führen Ergänzungen zum bestehenden Kurzarbeitsregelwerk zu den gewünschten positiven Beschäftigungseffekten, sobald die BIP-Wachstumsrate unter 0,5\% im Quartal liegt. In Expansionen nahmen dagegen kleine, weniger produktive Betriebe Kurzarbeit in Anspruch. Somit bewirken diskretionäre Kurzarbeitsmaßnahmen in Expansionen, dass unrentable Firmen durch die Lohnsubventionen künstlich am Leben gehalten werden.

\footnotetext{
4 Beispiele sind das Gesetz zur Sicherung von Beschäftigung und Stabilität in Deutschland und das folgende Beschäftigungschancengesetz, das für die Zeit vom 1. Februar 2009 bis 31. März 2012 vereinfachte Zugangsvoraussetzungen zum Bezug von Kurzarbeitergeld schaffte. Ein weiteres Beispiel ist die Verordnungsänderung des Bundesministeriums für Arbeit und Soziales, die die Bezugsfrist für das konjunkturelle Kurzarbeitergeld in der Zeit vom 1. Januar 2009 bis zum 31. Dezember 2009 auf 24 Monate verlängerte.

5 Kurzarbeit ermöglicht eine Beschäftigungsanpassung ohne Entlassungen und reduziert somit die Lohnsumme eines Betriebs, was dem Unternehmen zu mehr finanziellem Spielraum verhilft.
} 


\section{Kurzarbeit hat in der Großen Rezession den Arbeitsmarkt stabilisiert}

Zusammengenommen sicherten die regelgebundene und die diskretionäre Komponente der Kurzarbeit bis zu 850000 Jobs in der großen Rezession. Auf Basis des starken Rückgangs der gesamtwirtschaftlichen Leistung und des Anstiegs der Arbeitslosigkeit in früheren Rezessionen, wäre aber ein deutlich stärkerer Rückgang der Beschäftigung zu erwarten gewesen (siehe auch Balleer et al, 2016). Folglich waren andere Instrumente und Mechanismen ebenfalls von Bedeutung, wobei die Rolle und jeweilige Wichtigkeit arbeitsmarktpolitischer Maßnahmen wie Arbeitszeitkonten, Lohnzurückhaltung oder der Hartz-Reformen bis heute wissenschaftlich äußerst umstritten sind.

\section{Autonomes Fahren statt Stop and Go}

Eine Ausweitung des Zugangs zu Kurzarbeit in tiefen Rezessionen rettet zusätzliche Arbeitsplätze, während sie in normalen Konjunkturphasen relativ ineffektiv oder sogar kontraproduktiv ist. Vor diesem Hintergrund schlägt das BMAS vor, den Zugang zur Kurzarbeit bei Bedarf per Verordnungsermächtigung zu erleichtern.

Dieser Vorschlag erscheint auf den ersten Blick reizvoll, da das Ministerium auf Basis einer Vielzahl von Indikatoren und Informationen entscheiden kann, in welcher konjunkturellen Lage sich die Volkswirtschaft befindet und dann bei Bedarf ad hoc Kurzarbeit ausweiten kann. Bei genauerer Betrachtung birgt der Ministerialvorschlag allerdings zwei Tücken: Erstens besteht die Gefahr, dass Kurzarbeit gegen strukturellen Wandel eingesetzt wird. Zweitens wissen Unternehmen zwar, dass Kurzarbeit in Rezessionen vermutlich ausgeweitet wird. Es besteht aber ein hohes Maß an Unsicherheit wie und wann das passiert.

Die empirisch stark positiven Effekte der Ausweitung der Kurzarbeit in tiefen Rezessionen (Gehrke und Hochmuth 2019) basieren auf einem Umfeld, in dem Kurzarbeit ausschließlich konjunkturell eingesetzt wurde (insbesondere in der Großen Rezession). Die Literatur gibt Hinweise darauf, dass strukturelle Kurzarbeit wesentlich stärkere Nebenwirkungen hat als konjunkturelle Kurzarbeit. Boeri und Brücker (2011) verweisen darauf, dass Kurzarbeit in Italien eher strukturell eingesetzt wird und dort wesentlich weniger effektiv ist. Cooper et al. (2017) argumentieren, dass Kurzarbeit die optimale Allokation auf dem Arbeitsmarkt über den Konjunkturzyklus behindern kann. Aus unserer Sicht besteht gerade bei einer strukturellen Nutzung von Kurzarbeit die Gefahr, dass Arbeitskräfte an unproduktive Unternehmen gebunden werden und produktive Firmen unter 
Umständen nicht im gewünschten Umfang wachsen können. Somit kommt es zu einer ineffizienten Allokation von Arbeit in der Volkswirtschaft. Diese Ineffizienz wird potentiell verstärkt, wenn die Arbeitslosigkeit sehr niedrig ist, wie es, vor allem für Fachkräfte in Deutschland zurzeit der Fall ist.

Die aktuellen Vorschläge des BMAS zur Ausweitung von Kurzarbeit (siehe z. B. Heil, 2019) beziehen sich zwar auf die positiven Erfahrungen dieses Arbeitsmarktinstrumentes in der Großen Rezession. Die bisherige mediale Berichterstattung zum geplanten „Arbeit von Morgen“-Gesetz gibt aber zumindest Hinweise, dass eine stärkere strukturelle Nutzung der Kurzarbeit angedacht ist, z. B. im Kontext der Digitalisierung oder der Energiewende (siehe Roßbach, 2019). In unserem kürzlich erschienenen Artikel (Balleer et al. 2019) schlagen wir stattdessen vor, den Zugang zur Kurzarbeit in schweren Rezessionen automatisch auszuweiten. Dies reduziert die Gefahr, dass der großzügigere Zugang durch den strukturellen Wandel getrieben wird. Eine automatisierte Ausweitung der Kurzarbeit in schweren Rezessionen hätte darüber hinaus den großen Vorteil, dass Unternehmen entsprechende Änderungen einplanen können. Balleer et al. (2016) zeigen, wie wichtig Erwartungen für die Effektivität der Kurzarbeit sind. Eine Ausweitung der Kurzarbeit in Rezessionen auf Basis eines ministeriellen Erlasses verringert dagegen die Planbarkeit, wann und in welchem Umfang Kurzarbeit ausgeweitet wird, und verringert so die makroökonomische Effektivität des Instrumentes.

In einem Briefwechsel äußerte sich das BMAS uns gegenüber skeptisch, ob die von uns vorgeschlagene automatische Ausweitung des Zugangs in schweren Rezessionen implementierbar sei, da die Auswahl eines geeigneten Indikators schwierig und eine Ermittlung nur verzögert möglich sei. Es ist in der Tat richtig, dass eine Verwendung des Bruttoinlandsproduktes als Rezessionsindikator sich ausschließt, da dieses erst deutlich verzögert veröffentlicht wird und zudem starken Revisionen unterliegt. Zur zeitnahen Implementierung der Kurzarbeitsausweitungen stehen jedoch eine Vielzahl von Frühindikatoren, Prognosen und geschätzten Rezessionswahrscheinlichkeiten zur Verfügung, die wissenschaftlich hinsichtlich ihrer Eignung für eine stärkere Regelgebundenheit der Kurzarbeit bewertet werden sollten.

Zur Illustration vergleichen wir den Rezessionsindikator des strukturellen zeitvariierenden Vektorautoregressiven Modells aus Gehrke und Hochmuth (2019), einen zentrierten gleitenden Mittelwert aus fünf Quartalen BIP-Wachstum mit einem weit verbreiteten Frühindikator, dem ifo-Geschäftsklima. ${ }^{6}$ Abbildung 2

6 Wir verwenden bis 1990 das westdeutsche ifo-Geschäftsklima für das verarbeitende Gewerbe und ab 1991 das gesamtdeutsche Geschäftsklima für die gewerbliche Wirtschaft. Das Geschäftsklima basiert auf der ifo Konjunkturumfrage und ist ein transformierter Mittelwert aus den Salden der Geschäftslage und den Erwartungen. 


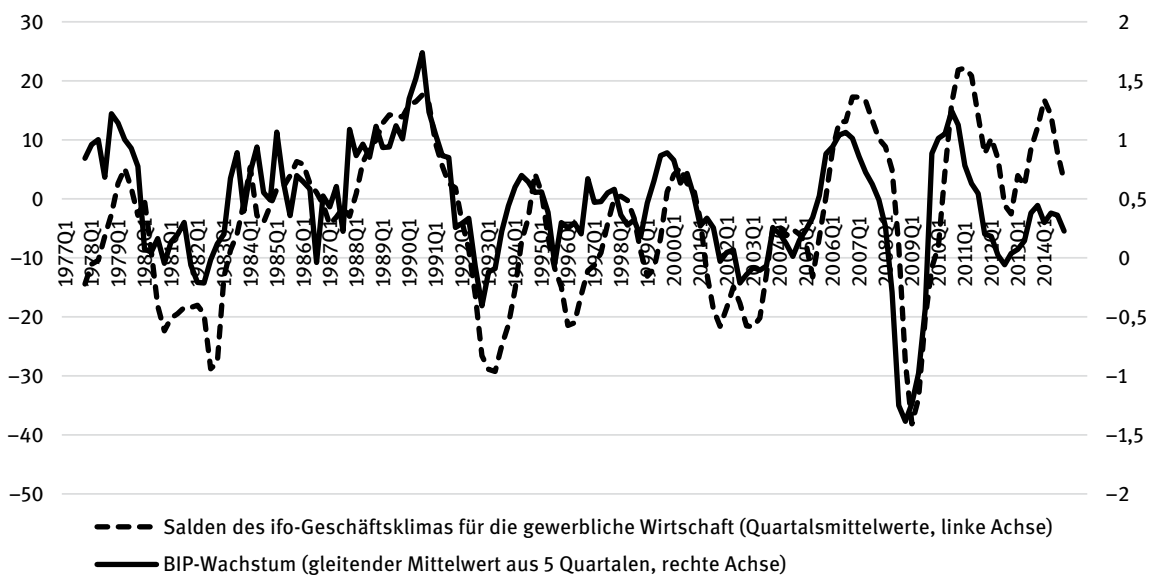

Abbildung 2: Vergleich des Rezessionsindikators von Gehrke und Hochmuth (2019) und den Salden des ifo-Geschäftsklimas für das verarbeitende Gewerbe bzw. die gesamte gewerbliche Wirtschaft (saisonbereinigt), 1977Q1-2014Q4 (Beobachtungszeitraum wie in Gehrke und Hochmuth, 2019).

Datenquelle: Destatis und ifo Institut.

zeigt, dass dieser in die Zukunft gerichtete Indikator über den gesamten Zeitraum eine deutliche Korrelation von 0,64 mit dem Rezessionsindikator aus Gehrke und Hochmuth (2019) aufweist. Zudem wird die Große Rezession sehr gut getroffen. Das deutet darauf hin, dass gesamtwirtschaftliche Frühindikatoren geeignete Zeitpunkte identifizieren können, in denen eine Ausweitung der Kurzarbeit zu positiven Beschäftigungseffekten führen kann.

\section{Zusammenfassung}

Zusammenfassend lässt sich sagen, dass Kurzarbeit den Arbeitsmarkt bei konjunkturellen Eintrübungen effektiv stabilisieren kann. Das existierende System verfügt bereits über eine regelgebundene Komponente, die erfolgreich stabilisiert. Hinzu kommen diskretionäre Änderungen, die in tiefen Rezessionen weitere Stabilisierungswirkungen entfalten. Wir schlagen vor, diese diskretionäre Komponente in das Regelwerk aufzunehmen und an entsprechenden Frühindikatoren zu orientieren. Dies würde die gesamtwirtschaftlichen Stabilisierungseffekte der Kurzarbeit weiter erhöhen. Darüber hinaus reduziert eine stärkere Regelbindung die Gefahr, dass Kurzarbeit strukturell oder zum falschen Zeitpunkt eingesetzt wird. 


\section{Literatur}

Balleer, Almut, Britta Gehrke, Wolfgang Lechthaler und Christian Merkl (2016). Does Short-Time Work Save Jobs: A Business Cycle Analysis? European Economic Review, 84, 99-122.

Balleer, Almut, Britta Gehrke, Brigitte Hochmuth und Christian Merkl (2019). Mit Kurzarbeit erfolgreich durch die nächste Rezession? Ifo Schnelldienst, 18/2019, S. 13-15.

Boeri, T. und Herbert Brücker (2011). Short-Time Work Benefits Revisited: Some Lessons from the Great Recession. Economic Policy, Oktober 2011, S. 697-765.

Cooper, R., Meyer, M., Schott, I. (2017): The Employment and Productivity Effects of Short-Time Work in Germany. NBER Working Paper, No. 23688.

Gehrke, Britta und Britta Hochmuth (2019). Counteracting Unemployment in Crises: Non-linear Effects of Short-Time Work Policy. Scandinavian Journal of Economics, im Erscheinen.

Heil, Hubertus (2019). Die wichtigste Ressource bleibt der Mensch. Ifo Schnelldienst, 18/2019, S. 7-9.

Roßbach, Henrike (2019). Was die Metallarbeitgeber an Heils Qualifizierungs-Gesetz ablehnen. Süddeutsche Zeitung vom 17. September 2019 (www.sueddeutsche.de/wirtschaft/arbeitvon-morgen-gesetz-heil-1.4602574, letzter Zugriff am 13. November 2019). 